\title{
CLC-2 is a positive modulator of oligodendrocyte precursor cell differentiation and myelination
}

\author{
XIAOLIN HOU ${ }^{1 *}$, RUI ZHANG ${ }^{2 *}$, JUNYAN WANG $^{2 *}$, YUNHONG LI $^{2 *}$, FAN LI $^{2}$, YAN ZHANG $^{2}$, \\ XIAOMIN ZHENG ${ }^{2}$, YING $\mathrm{SHEN}^{3}$, YIN WANG ${ }^{2}$ and LIANG ZHOU ${ }^{3}$ \\ ${ }^{1}$ Department of Neurology, General Hospital of Ningxia Medical University; \\ ${ }^{2}$ Ningxia Key Laboratory of Cerebrocranial Diseases, Basic Medical School of Ningxia Medical University, \\ Yinchuan, Ningxia 750004; ${ }^{3}$ Department of Neurobiology, Zhejiang University School of Medicine, \\ Hangzhou, Zhejiang 310058, P.R. China
}

Received September 12, 2017; Accepted December 18, 2017

DOI: $10.3892 / \mathrm{mmr} .2018 .8439$

\begin{abstract}
Oligodendrocytes (OLs) are myelin-forming cells that are present within the central nervous system. Impaired oligodendrocyte precursor cell (OPC) differentiation into mature OLs is a major cause of demyelination diseases. Therefore, identifying the underlying molecular mechanisms of OPC differentiation is crucial to understand the processes of myelination and demyelination. It has been acknowledged that various extrinsic and intrinsic factors are involved in the control of OPC differentiation; however, the function of ion channels, particularly the voltage-gated chloride channel (CLC), in OPC differentiation and myelination are not fully understood. The present study demonstrated that CLC-2 may be a positive modulator of OPC differentiation and myelination. Western blotting results revealed that CLC-2 was expressed in both OPCs and OLs. Furthermore, CLC-2 currents $\left(\mathrm{I}_{\mathrm{CLC}-2}\right)$ were recorded in both types of cells. The inhibition of $\mathrm{I}_{\mathrm{CLC}-2}$ by GaTx2, a blocker of CLC-2, was demonstrated to be higher in OPCs compared with OLs, indicating that CLC-2 may serve a role in OL differentiation. The results of western blotting and immunofluorescence staining also demonstrated that the expression levels of myelin basic protein were reduced following GaTx 2 treatment, indicating that the differentiation
\end{abstract}

Correspondence to: Professor Liang Zhou, Department of Neurobiology, Zhejiang University School of Medicine, 866 Yu Hang Tang, Hangzhou, Zhejiang 310058, P.R. China E-mail:zllzlj@zju.edu.cn

Professor Yin Wang, Ningxia Key Laboratory of Cerebrocranial Diseases, Basic Medical School of Ningxia Medical University, 1160 Shengli Street, Yinchuan, Ningxia 750004, P.R. China

E-mail: yin-wang@hotmail.com

*Contributed equally

Key words: voltage-gated chloride channel 2, differentiation, oligodendrocyte precursor cells, oligodendrocytes, myelination, GaTx2 of OPCs into OLs was inhibited following CLC-2 inhibition. In addition, following western blot analysis, it was also demonstrated that the protein expression of the myelin proteins yin yang 1, myelin regulatory factor, Smad-interacting protein 1 and sex-determining region Y-box 10 were regulated by CLC-2 inhibition. Taken together, the results of the present study indicate that CLC-2 may be a positive regulator of OPC differentiation and able to contribute to myelin formation and repair in myelin-associated diseases by controlling the number and open state of CLC-2 channels.

\section{Introduction}

In the central nervous system (CNS), myelin forms a medullary sheath around the membrane of axons and is developed by oligodendrocytes (OLs). Adult OLs are derived from oligodendrocyte precursor cells (OPCs) (1). The survival and differentiation of OLs is essential in axon myelination and myelin repair in diseases of the CNS that are caused by demyelination, which include multiple sclerosis, periventricular leukomalacia and adrenoleukodystrophy (2-5). Investigation of the underlying molecular mechanisms that regulate OPC differentiation is important in understanding myelin formation and repair in demyelination disorders.

Advances have been made towards understanding how the OPC differentiation process is controlled by various extrinsic and intrinsic factors (6,7). Axon-derived ligands, including Jagged/Notch (8), polysialylated-neural adhesion molecule (9) and leucine-rich repeat and immunoglobulin-like domain-containing protein 1 (10), have been reported to prevent OL differentiation and/or myelination. Transcription factors such as OL transcription factor (Olig)1, Olig2, myelin regulatory factor (MRF), sex-determining region Y-box (Sox)10, Sox2, Sox6, inhibitor of DNA binding (Id)-2, Id4, yin yang 1 (YY1) and Smad-interacting protein 1 (Sip1) function in the maintenance of an undifferentiated state in OPCs as they suppress myelin gene expression that is required for the generation of mature, post-mitotic OLs (11-16). Wnt/ $\beta$-catenin signaling has been reported to be a major regulator of $\mathrm{OL}$ development (17). Our previous study also demonstrated that glycogen synthase kinase 3â may promote the differentiation 
of OPCs through a â-catenin-mediated mechanism of transcriptional regulation (18).

There are very few reports regarding the roles of ion channels in OL differentiation and myelination, although numerous types of ion channels are expressed in OPCs and OLs. Williamson et al (19) identified voltage-activated tetrodotoxin-sensitive $\mathrm{Na}^{+}$currents, two classes of voltage-activated outward $\mathrm{K}^{+}$currents, an inactivating inwardly rectifying $\mathrm{K}^{+}$ current, a voltage-activated $\mathrm{Cl}^{-}$current and three classes of $\mathrm{Ca}^{2+}$ currents in OL progenitors. Furthermore, an inward rectifying potassium channel (Kir4.1)-specific antibody was detected in children with acquired demyelinating CNS diseases (20), and Kir4.1 has been reported to be abnormally expressed in focal areas of patients with multiple sclerosis (21). Additionally, $\mathrm{Ca}^{2+}$ transients are considerably larger in mature OLs compared with OPCs, indicating that $\mathrm{Ca}^{2+}$ may be a signal for the initiation of myelin formation (22). The inhibition of voltage-gated chloride channel (CLC)-2, by 4,4'-diisothiocyanostilbene2,2'-disulfonic acid (DIDS), is able to protect developing OLs in the white matter following ischemia-hypoxia injury in neonatal rats (23). However, the function of CLC-2 on OPC differentiation and myelination is not fully understood.

In the present study, the results demonstrated that CLC-2 may serve important roles in OPC development and differentiation. The development of OPCs may be inhibited by treatment with the CLC-2 specific inhibitor GaTx2. In addition, the inhibition of CLC-2 affected expression of myelin-associated proteins and may be involved in myelin development in the CNS; if CLC-2 gene was mutant or CLC-2 channel was blocked, myelin-associated proteins would be inhibited, and myelin formation would be affected.

\section{Materials and methods}

Ethics statement. Animals were treated according to the standards of the Animal Care and Use Committee of Zhejiang University. All procedures in the present study were approved by the Ethics Committee of Zhejiang University (Hangzhou, China).

Chemicals. Antibodies against CLC-2 (cat. no. sc-20122), YY1 (cat. no. sc-7341), Sip1 (cat. no. sc-48789) and MRF (cat. no. sc-137607) were purchased from Santa Cruz Biotechnology, Inc. (Dallas, TX, USA). The antibody against platelet-derived growth factor (PDGF) $\alpha$ receptor (PDGF $\alpha$ R; cat. no. 558774) was obtained from BD Biosciences (Franklin Lakes, NJ, USA). Antibodies against myelin basic protein (MBP; cat. no. AB5864), Olig2 (cat. no. AB109186), myelin-associated glycoprotein (MAG; cat. no. ST1684), 2',3'-cyclic nucleotide 3'-phosphodiesterase (CNP; cat. no. MAB326), Sox2 (cat.no.AB5603), Sox10 (cat.no. AB5727) and GAPDH (cat. no. CB1001) were purchased from EMD Millipore (Billerica, MA, USA). The marker of proliferation Ki67 (Ki67) antibody (cat no. ab15580) was obtained from Abcam (Cambridge, UK). Triiodothyronine (T3) was from Sigma-Aldrich (Merck KGaA, Darmstadt, Germany). Dulbecco's modified Eagle's medium (DMEM), neurobasal medium, B27 supplements, proteinase inhibitor cocktail, horseradish peroxidase-conjugated secondary antibodies for western blotting,DAPI and Alexa Fluor-conjugated secondary antibodies (Goat anti-Rabbit IgG $(\mathrm{H}+\mathrm{L})$ Cross-Adsorbed Secondary Antibody, Alexa Fluor 568, cat no. A-11011; Goat anti-Rabbit IgG (H+L) Cross-Adsorbed Secondary Antibody, Alexa Fluor 488, cat no. A-11008; Goat anti-Rat IgG (H+L) Cross-Adsorbed Secondary Antibody, Alexa Fluor 555, cat no. A-21434) were from Invitrogen (Thermo Fisher Scientific, Inc., Waltham, MA, USA). GaTx2 was purchased from Smartox Biotechnology (Grenoble, France). Recombinant PDGF AA (cat. no. AF 100 13A) was purchased from PeproTech, Inc. (Rocky Hill, NJ, USA). Bicinchoninic acid and enhanced chemiluminescence (ECL) kits were from Pierce (SuperSignal ${ }^{\text {TM }}$ West Femto Maximum Sensitivity Substrate; cat. no. 34096; Thermo Fisher Scientific, Inc.). All other chemicals were purchased from OriGene Technologies, Inc. (Rockville, MD, USA) unless stated otherwise.

OPC culture. Primary OPC cultures were modified from a previous publication (24) in which postnatal day 2-4 CD rats were dissected out, minced and digested for $15 \mathrm{~min}$ at $37^{\circ} \mathrm{C}$ in S-MEM. In the present study, postnatal day 1-3 rat cortices were digested for $20 \mathrm{~min}$ at $37^{\circ} \mathrm{C}$ in DMEM. Cortical glial cell mixtures were obtained from 10 newborn Sprague Dawley rats provided by Animal Center of Zhejiang University, Hangzhou, China ( $\mathrm{n}=80$, male, passage $1-3$, weight, 5-10 g). Rats were kept in temperature and humidity-controlled conditions $\left(22^{\circ} \mathrm{C}\right.$, 55-60\% humidity) under 12-h light/dark cycle with food and water available ad libitum. Cells from 10 rats $\left(5 \times 10^{5}\right.$ cells per flask) were seeded into five $75 \mathrm{~cm}^{2}$ tissue culture flasks in DMEM with $20 \%$ fetal bovine serum (cat. no. 12483012; Thermo Fisher Scientific, Inc.) for 10 days at $37^{\circ} \mathrm{C}$ and $5 \%$ $\mathrm{CO}_{2}$. The culture medium was replaced every 3 days. Cultures were shaken for 1-2 $\mathrm{h}$ at $200 \mathrm{rpm}$ to remove microglia, incubated in fresh DMEM medium with $20 \%$ fetal bovine serum for $4 \mathrm{~h}$ and subsequently shaken at $260 \mathrm{rpm}$ at $37^{\circ} \mathrm{C}$ for $16-18 \mathrm{~h}$ for OPC collection. Detached cell suspension was collected and left to adhere in uncoated Petri dishes for $1 \mathrm{~h}$ at $37^{\circ} \mathrm{C}$ and $5 \% \mathrm{CO}_{2}$, which allowed microglia and astrocytes to firmly attach, while OPCs were loosely attached and were collected by gently shaking the dishes. Collected OPCs were re-plated at $5 \times 10^{3}-5 \times 10^{4}$ cells $/ \mathrm{cm}^{2}$ onto poly-D-lysine-coated plates, dishes or coverslips in neurobasal medium supplemented with $2 \%$ B27. To expand OPCs and to keep them undifferentiated, the culture medium was supplemented with PDGF-AA (10 nM). For differentiation assays, OPCs were cultured in a differentiation medium (neurobasal medium containing $40 \mathrm{ng} / \mathrm{ml} \mathrm{T} 3$ and $2 \% \mathrm{~B} 27)$ for 3 days.

Treatment of cells by GaTx2. OLs or OPCs were seeded at $5 \times 10^{4}$ cells- $1 \times 10^{5}$ cells/well. To expand OPCs and to keep them undifferentiated, the culture medium was supplemented with PDGF-AA (10 nM) for 4 days. For differentiation assays, OPCs were cultured in medium with PDGF-AA (10 nM) for 1 day, then changed with medium containing $40 \mathrm{ng} / \mathrm{ml} \mathrm{T3}$ for another 3 days. For the control, only T3 was added, but for the experimental groups, T3 together with 10, 5, 2, 1, 0.2 or $0.1 \mathrm{nM}$ GaTx2 were added for different time points (12, 24 and $72 \mathrm{~h}$ ).

Immunocytochemistry. OLs or OPCs were seeded at $5 \times 10^{4}$ cells in $100 \mu \mathrm{l} /$ well on coverslips in 24 -well plates. For immunostaining, cells were washed twice with PBS, fixed with 
4\% paraformaldehyde in PBS for $15 \mathrm{~min}$ at room temperature, washed three times with PBS and preincubated in $10 \%$ bovine serum albumin (cat. no. A8806; Sigma-Aldrich; Merck KGaA) and $0.4 \%$ Triton X-100 in PBS for $1 \mathrm{~h}$ at room temperature, prior to being immediately exposed to primary antibodies overnight at $4^{\circ} \mathrm{C}$. The primary antibodies used for immunostaining were anti-PDGF $\alpha$ R (1:500), anti-MBP (1:5,000), anti-Olig2 (1:1,000) and anti-Ki67 (1:1,000). Cultures were rinsed in PBS and incubated with fluorophore-conjugated secondary antibodies for $1 \mathrm{~h}$ at room temperature. The sections were mounted using ProLong Gold Antifade Reagent with DAPI (cat. no. P36931; Invitrogen; Thermo Fisher Scientific, Inc.) and immediately covered with coverslips. Immunocytochemical images were obtained with a fluorescence microscope (magnification, $\mathrm{x} 20$; Olympus Corporation, Tokyo, Japan) at a resolution of $1,360 \times 1,024$ pixels. A total of 10 fields were analyzed with Image J software version $1.49 \mathrm{v}$ (National Institutes of Health, Bethesda, MD, USA). The parameters used for microscopy were consistent in all experiments.

Western blotting. To determine the expression of certain proteins, OPC and OLs cultures were lysed in radioimmunoprecipitation assay buffer (cat. no. P0013C; Beyotime Institute of Biotechnology, Shanghai, China) containing a proteinase inhibitor cocktail and harvested. Following incubation at $4^{\circ} \mathrm{C}$ for $30 \mathrm{~min}$, the lysates were centrifuged at $8,050 \mathrm{xg}$ at $4^{\circ} \mathrm{C}$ for $30 \mathrm{~min}$. Protein concentrations were measured in each sample using a BCA assay. Protein denaturation was subsequently performed in a sample buffer containing 2-mercapto ethanol and bromophenol blue for $10 \mathrm{~min}$ at $95^{\circ} \mathrm{C}$. Equal quantities of protein $(10 \mu \mathrm{g})$ were resolved by $10 \%$ SDS-PAGE and transferred onto polyvinylidene difluoride membranes (EMD Millipore). The membranes were blocked with 5\% non-fat milk in TBS containing $0.1 \%$ Tween-20 for $1 \mathrm{~h}$ at room temperature and incubated at $4^{\circ} \mathrm{C}$ overnight with the indicated antibodies (anti-CLC-2, 1:200; anti-MBP, 1:5,000; anti-CNP, 1:10,000; anti-MAG, 1:1,000; anti-YY1, 1:500; anti-MRF, 1:1,000; anti-Sip1, 1:2,000; anti-Sox 2, 1:2,000; and, anti-Sox 10, 1:1,000). After washing with PBS, membranes were incubated with secondary antibodies at dilutions of 1:10,000 at room temperature for $1 \mathrm{~h}$. The proteins were subsequently visualized using the ECL kit and membranes were exposed to X-ray films. The blotting signals were digitally scanned and quantified using ImageJ version $1.49 \mathrm{v}$ software (National Institutes of Health, Bethesda, MD, USA). The immunoreactivity of GAPDH $(1: 2,000)$ was set as the loading control. All data were normalized to the corresponding control.

Electrophysiology. Whole-cell currents in OPCs and OLs were measured using tight-seal whole-cell voltage-clamp techniques, as previously described (25). Data acquisition and command potentials were controlled by pCLAMP version 10.0 software (Molecular Devices, LLC, Sunnyvale, CA, USA). Whole-cell currents were filtered at $1 \mathrm{kHz}$ and sampled at $5 \mathrm{kHz}$. Recording pipettes were prepared from borosilicate glass electrodes (1.5 $\mathrm{mm}$ outside diameter) with tip resistance of 1-3 M $\Omega$ when filled with pipette solutions. After a tight seal between the cell membrane and the pipette tip (seal resistance $>10 \mathrm{G} \Omega$ ) had been formed, the membrane patch was ruptured with brief additional suction. The capacitive transients elicited by symmetrical $5-\mathrm{mV}$ steps from $0 \mathrm{mV}$ were recorded at $100 \mathrm{kHz}$ for subsequent calculation of capacitance and access resistance. Input resistance was defined as the amplitude of the steady state voltage response divided by the current amplitude, and input conductance as the reciprocal value of input resistance. To account for differences in cell size, whole-cell currents were normalized to cell capacitance and the average data were reported as current densities $(\mathrm{pA} / \mathrm{pF})$. To isolate CLC-2 currents, cells were perfused with a $\mathrm{Na}^{+}$- and $\mathrm{K}^{+}$-free extracellular solution containing $145 \mathrm{mM} \mathrm{NaCl}, 3 \mathrm{mM} \mathrm{KCl}$, $2 \mathrm{mM} \mathrm{CaCl}_{2}, 10 \mathrm{mM}$ HEPES and $10 \mathrm{mM}$ glucose [310 osmole (Osm); pH 7.4 adjusted with $\mathrm{NaOH}$. The pipette solution contained an intracellular solution containing $135 \mathrm{mM} \mathrm{Cs}$ methansulfonate, $10 \mathrm{mM} \mathrm{CsCl}, 0.2 \mathrm{mM}$ EGTA, $10 \mathrm{mM}$ HEPES, 4 mM Na-ATP and 0.4 mM Na-GTP (310 Osm; pH adjusted to 7.3 with $\mathrm{KOH}$ ). To elicit CLC-2 currents, voltage steps from +40 up to $-140 \mathrm{mV}$ from a holding potential of $0 \mathrm{mV}$ were used. A final $50 \mathrm{msec}$ voltage step at $+40 \mathrm{mV}$ was applied before returning to the holding potential. Cell cultures were stimulated with voltage pulses between -140 and $+60 \mathrm{mv}$ and the currents were recorded as the control. Experiments were conducted at room temperature. Averaging, normalizing and subtracting trace profiles were performed off-line using Clamp Fit version 10.2 (Mapu Scientific LLC, Guangzhou, China). For trace subtraction, current traces recorded from GaTx2 application were subtracted ('Arithmetic tool') from the respective currents from control condition to examine CLC-2-specific current/capacitance profiles in OPCs and OLs.

Statistical analysis. Data analysis was performed using Excel 2003 (Microsoft Corporation, Redmond, WA, USA), Igor Pro version 6.0 (Wave Metrics, Lake Oswego, OR, USA), and SPSS version 16.0 (SPSS, Inc., Chicago, IL, USA). Statistical differences were determined by Student's t-test for two-group comparisons or one-way analysis of variance followed by Tukey's post-hoc test for multiple comparisons. $\mathrm{P}<0.05$ was considered to indicate a statistically significant difference. Data in the text and figures are presented as the mean \pm standard error of the mean.

\section{Results}

Assessment of cultured OPCs and OLs. To examine the purity of OPCs and OLs, cells were immune stained with antibodies against PDGF $\alpha \mathrm{R}$, an OPC specific marker, and Olig2, which is expressed in both OLs and OPCs. As demonstrated in Fig. 1A, the purity of oligodendrocytes (OPCs+OLs) was $>85 \%$ (PDGF $\alpha \mathrm{R}+\mathrm{Olig} 2+\mathrm{DAPI}$ ). To assess the differentiation state induced by $\mathrm{T} 3$ treatment for 3 days, the expression levels of the mature OL marker protein, MBP, was determined. It was demonstrated that MBP was expressed primarily in mature OLs, while Olig2 was present in OPCs and OLs (Fig. 1B). These results indicated that $\mathrm{T} 3$-induced differentiation of OPCs was successful.

Effects of CLC-2 inhibitor GaTx2 on CLC-2 currents. The presence of CLC-2 protein in OPCs and OLs was initially determined. Western blotting revealed that CLC-2 was expressed in both OPCs and mature OLs (Fig. 2A). The effects of CLC-2 inhibition in OPCs and OLs were subsequently 
A
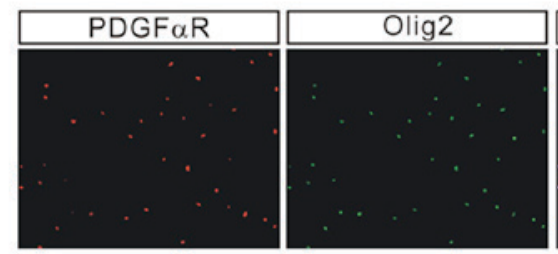

B

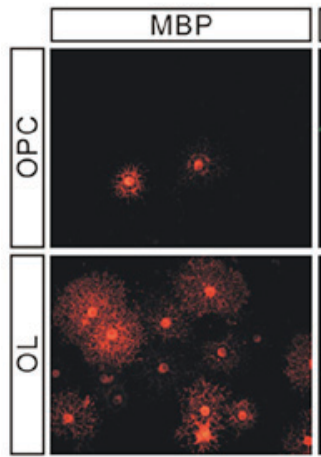

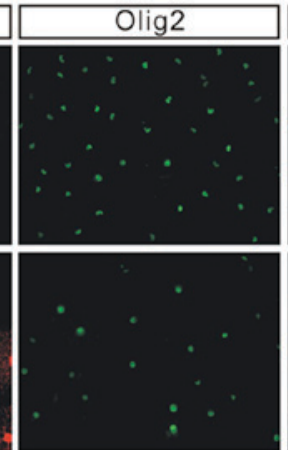

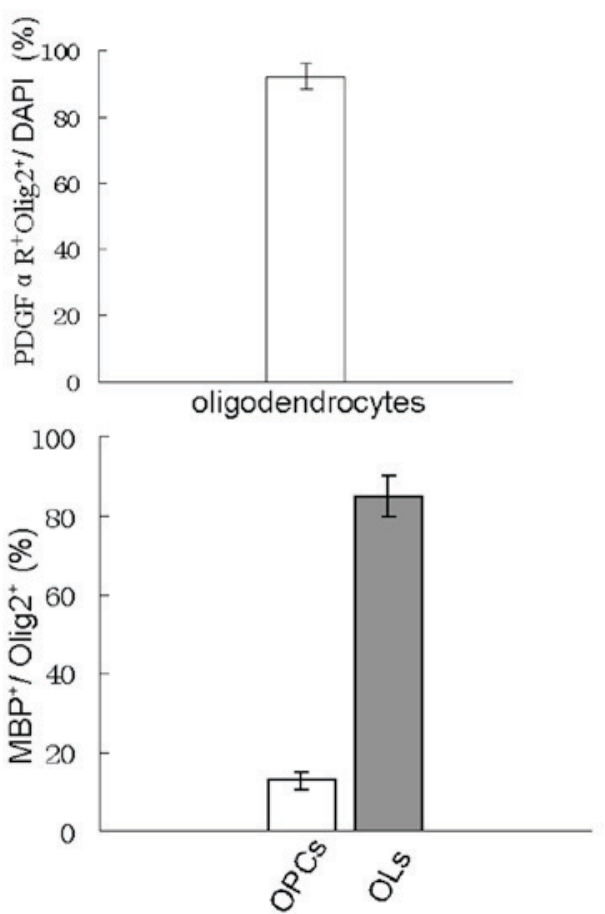

Figure 1. Assessment of cultured OPCs and OLs. (A) OPCs and OLs were immunostained with antibodies against PDGFaR and Olig2 to examine their expression levels in cultured cells. The purity of OPCs reached $>85 \%$. Scale bar, $40 \mu \mathrm{m}$. The purity of cultured OPCs was determined by the percentage of PDGF $\alpha \mathrm{R}^{+} \mathrm{Olig} 2^{+}$cells among DAPI-stained cells, as demonstrated in the bar graphs to the right $(\mathrm{n}=10)$. (B) Differentiation induced by T3 for 3 days as measure by MBP and Olig2 immunostaining. In OPCs, $\mathrm{MBP}^{+} / \mathrm{Olig} 2^{+}$is $13 \pm 2 \%$, but in OLs, $\mathrm{MBP}^{+} / \mathrm{Olig}^{+}$is $85 \pm 5 \%$. Scale bar, $20 \mu \mathrm{m}$. OPCs, oligodendrocyte precursor cells; OLs, oligodendrocytes; PDGF $\alpha$ R, platelet-derived growth factor $\alpha$ receptor; Olig2, oligodendrocyte transcription factor 2; T3, triiodothyronine MBP; myelin basic protein.

examined using electrophysiological experiments. Cell cultures were stimulated with voltage pulses between -140 and $+60 \mathrm{mv}$ and the currents were recorded as the control (Fig. 2B). $\mathrm{I}_{\mathrm{CLC}-2}$ were recorded in OPCs and OLs (left panels of Fig. 2C and D, respectively). GaTx2 (10 nM) was added to cells for $4 \mathrm{~h}$ to suppress CLC-2 activity. The alteration of currents in the control and GaTx2 treatment groups were presented in Fig. 2C and D. The left panels in Fig. 2C and D represented control CLC-2 currents. The middle panels represented the CLC-2 currents recorded after supplementing with $10 \mathrm{nM}$ GaTx2. The right panels represented the currents of the middle panels subtracted from the left panels. The results indicated that residual currents following inhibition of CLC-2 with GaTx 2 were higher in OLs compared with OPCs, indicating that CLC-2 may serve roles in OL differentiation. The curves of current and voltage association of OPCs and OLs were presented in Fig. 2E.

Effective concentration of GaTx 2 in the inhibition of OL activity. To determine the most effective concentration and duration of GaTx 2 treatment in OLs and OPCs, OL cultures were treated with different concentrations of GaTx2 $(0.1,0.2,1,2$ and $5 \mathrm{nM})$ for different durations (12, 24 and $72 \mathrm{~h}$ ). Western blot analysis demonstrated that MBP levels were decreased significantly at 12 and $24 \mathrm{~h}$ after 2 and $5 \mathrm{nM}$ GaTx 2 treatment compared with the control group, but no significant differences were observed at $72 \mathrm{~h}$ (Fig. 3A), which indicated that the effect of GaTx 2 may be sustained only for $24 \mathrm{~h}$. Therefore, the authors of the present study decided to add GaTx2 into cultured OPCs at the same concentration ( $2 \mathrm{nM}$ ) every $24 \mathrm{~h}$, and $40 \mathrm{ng} / \mathrm{ml} \mathrm{T3}$ was added for $72 \mathrm{~h}$ to induce differentiation. Immunofluorescence results demonstrated that following $5 \mathrm{nM}$ GaTx 2 treatment, the number of Olig2-positive cells decreased significantly compared with the control group, while $2 \mathrm{nM}$ GaTx 2 treatment had no effect on cell number, which indicated that $2 \mathrm{nM}$ GaTx 2 treatment had little effect on cell viability (Fig. 3B).

Effects of GaTx2 on OPC differentiation. As GaTx2 decreased CLC-2 currents in OPCs and OLs, it was investigated whether CLC-2 inhibition affected OPC differentiation by measuring the levels of myelin-associated proteins, including MBP, MAG and CNP. Control cells were treated with $10 \mathrm{nM}$ PDGF-AA for 1 day and $40 \mathrm{ng} / \mathrm{ml} \mathrm{T3}$ was added for 3 days to induce differentiation. The experimental group was treated with T3 together with $2 \mathrm{nM}$ GaTx 2 for $72 \mathrm{~h}$ and GaTx 2 was added every $24 \mathrm{~h}$. The results revealed that CLC-2 inhibition with $2 \mathrm{nM}$ GaTx 2 substantially decreased the expression levels of MBP, while CNP was unchanged, compared with the control group (Fig. 4A). Following 2 nM GaTx2 treatment, myelin related glycoprotein (MAG) expressed as dMAG, a form of lower molecular weight without a protein modication function. Since the expression of Ki67 protein is closely associated with cell proliferation (26), Ki67 immunostaining was used to determine whether GaTx2 affected the proliferation of OPCs. The immunocytochemical results demonstrated that GaTx2 treatment decreased the percentage of MBP-positive cells (Fig. 4B), but not Ki67-positive cells (Fig. 4C), which indicated that GaTx2 may inhibit OPC differentiation but had no effect 
A

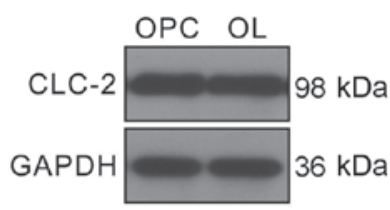

C

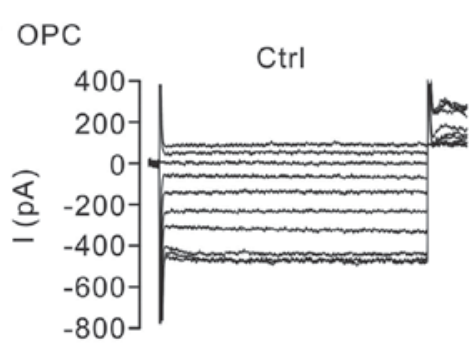

D
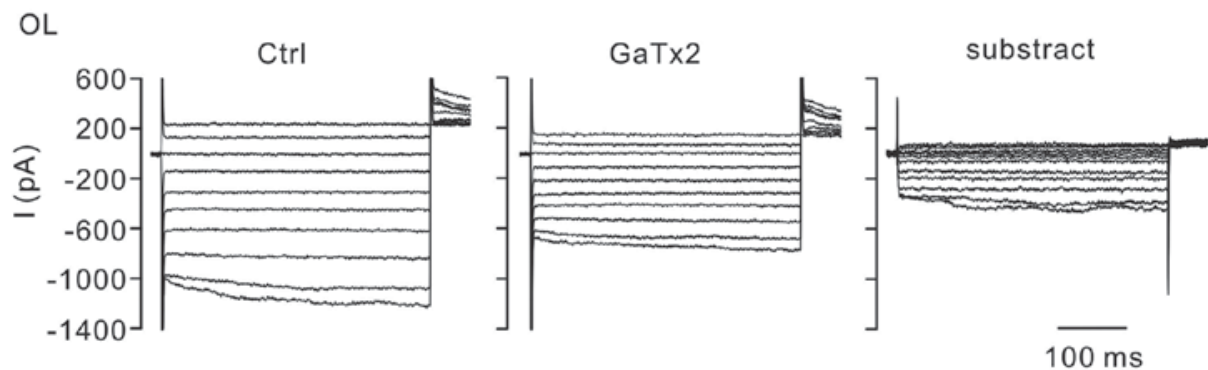

B

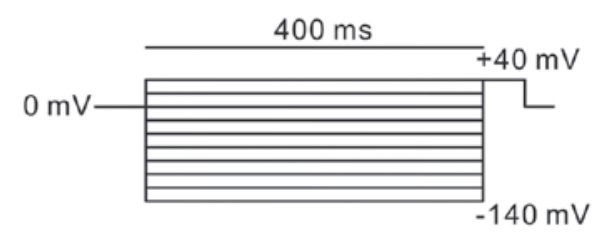

E

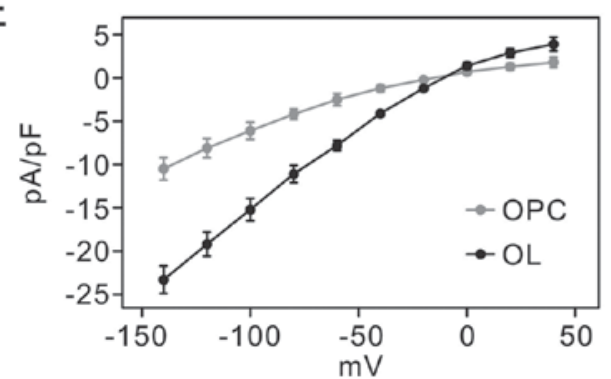

Figure 2. Effect of GaTx2 on CLC-2 current ( $\left.\mathrm{I}_{\mathrm{CLC}-2}\right)$ in OPCs and OLs. (A) CLC-2 protein expression levels in OPCs and OLs. GAPDH served as the internal control. Bands were analyzed with ImageJ version 1.49v software (National Institutes of Health, Bethesda, MD, USA). The densities of the bands were: CLC2 in OPC (779.07 \pm 21$)$, OL (772.44 \pm 19$)$, GAPDH in OPC (628.09 \pm 12$)$ and OL (631.48 \pm 9$)$. (B) Schematic diagram of voltage stimulation mode. (C) OPCs and (D) OLs were stimulated with voltages between -140 and $+60 \mathrm{mV}$ in the three groups. $\mathrm{I}_{\mathrm{CLC}-2}$ (right panel) was calculated by subtracting GaTx 2 (10 $\mathrm{nM}$ )-sensitive currents (middle panel) from the control current (left panel). (E) Current to voltage association curves of $\mathrm{I}_{\mathrm{CLC}-2}$ in OPCs and OLs. OPCs, oligodendrocyte precursor cells; OLs, oligodendrocytes; CLC-2, voltage-gated chloride channel 2.

on their proliferation. These data suggested that the inhibition of OPC differentiation by $2 \mathrm{nM}$ GaTx 2 is not mediated via altered cell survival or proliferation.

Effects of CLC-2 inhibition on transcriptional factors of $O L$ differentiation. The present study demonstrated that MBP was significantly decreased by GaTx 2 treatment. As an important myelin-associated protein in OLs, the expression of MBP is regulated by various transcriptional factors, including YY1, MRF, Sip1, Sox2 and Sox10. Therefore, the expression levels of several transcriptional factors following GaTx 2 treatment were also measured by western blotting. The results demonstrated that GaTx2 treatment decreased the levels of YY1, MRF, Sip1 and Sox10 proteins, which positively regulate MBP transcription (Fig. 5). However, the expression of Sox2, a negative transcriptional regulator of MBP expression, was unaltered (Fig. 5). These results indicated that inhibition of OPC differentiation by GaTx 2 may be due to reduced expression levels of these transcription factors.

\section{Discussion}

CLC-2 is broadly expressed on the plasma membrane of various types of cells in the brain and epithelia (27). In the 
A

GaTx2 (nM)
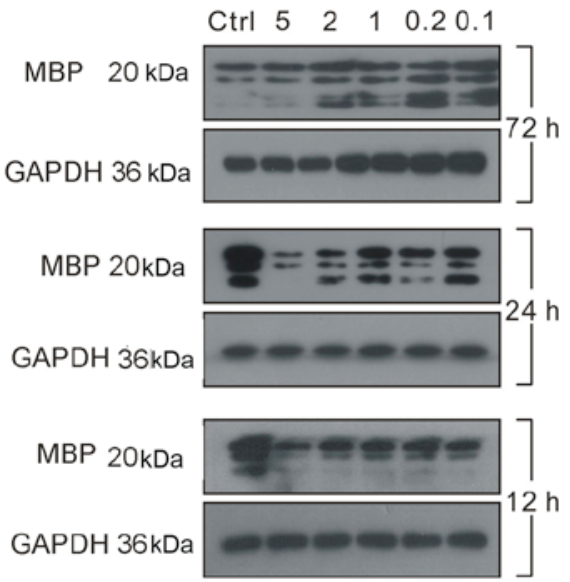

$12 \mathrm{~h}$

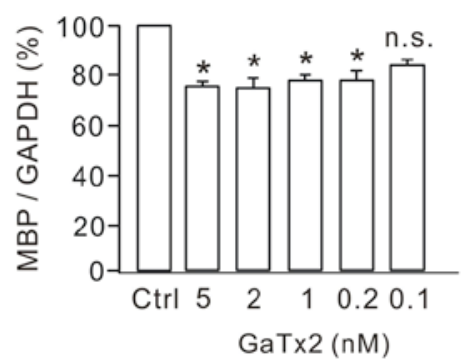

$\mathrm{GaT} \times 2(\mathrm{nM})$

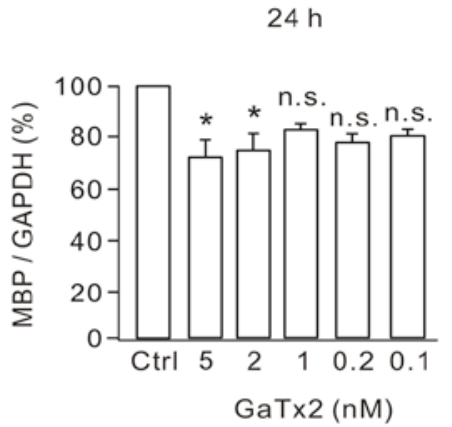

$72 \mathrm{~h}$

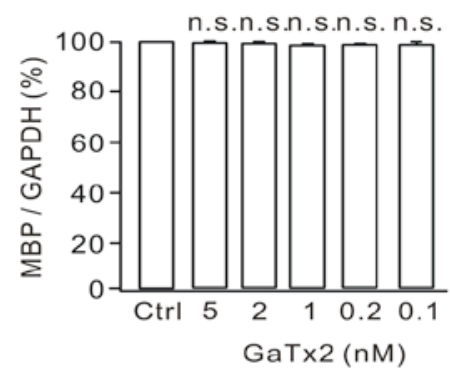

$24 \mathrm{~h}$
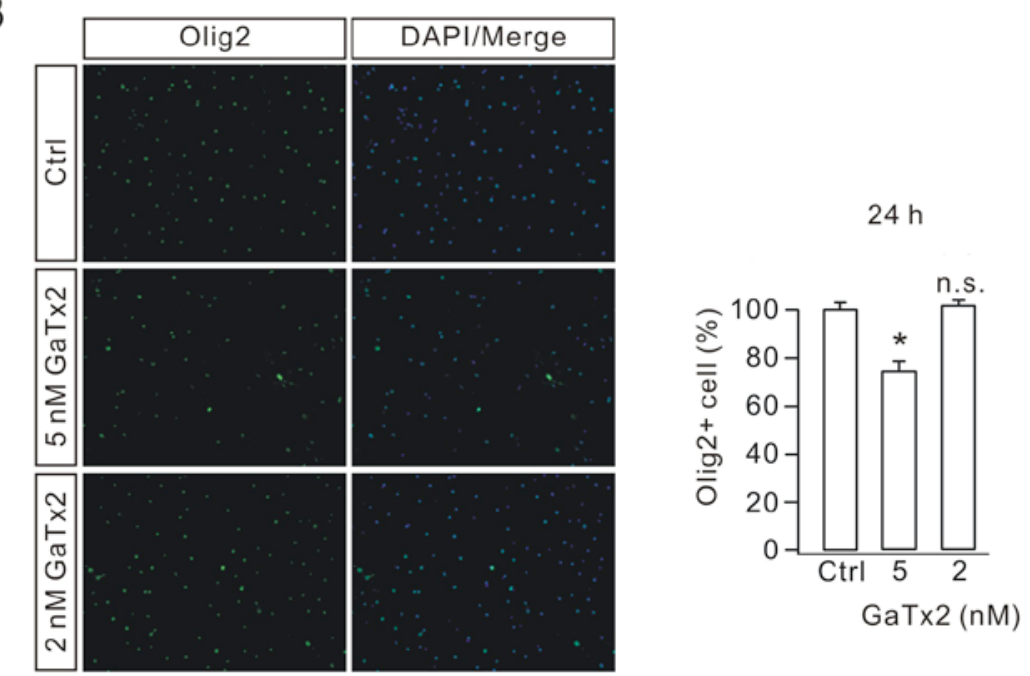

Figure 3. Effective concentration of GaTx2 in the inhibition of OL activity. (A) OL cultures were treated with different concentrations of GaTx2 for different durations. MBP expression levels were measured by western blotting and densitometric analysis was performed to quantify protein levels. (B) Olig2 immunostaining following 2 or $5 \mathrm{nM} \mathrm{GaTx} 2$ treatment for $24 \mathrm{~h}$, indicating the difference compared with the control group. The experiment was repeated four times. Scale bar, $40 \mu \mathrm{m}$. "P<0.05 vs. Ctrl. OLs, oligodendrocytes; MBP, myelin basic protein; Olig2, oligodendrocyte transcription factor 2; Ctrl, control; n.s., not significant.

CNS, CLC-2 is present on the endfeet of astrocytes and hippocampal neurons and is implicated in chloride homeostasis and $\mathrm{Cl}^{-}$movements (28). The present study demonstrated that functional CLC-2 channels were observed in OPCs and mature OLs, and may be associated with the differentiation of OPCs and myelin gene expression. This conclusion was corroborated by results that were obtained following the pharmacological inhibition of CLC-2 in the present study. It was also revealed that the effect of CLC-2 inhibition on OPC differentiation was not a result of decreased proliferation levels (as measured by $\mathrm{Ki} 67$ expression). By contrast, activation of CLC-2 was sufficient to promote the differentiation of OPCs. Finally, it was demonstrated in the present study that CLC-2 inhibition reduced the expression levels of the transcription factors YY1, MRF, Sox10 and Sip1, which are necessary for myelin protein expression $(7,13,29)$. The aforementioned results of the present study indicated that CLC-2 may positively regulate the differentiation of
OPCs through regulation of the transcription of OPC differentiation factors.

It has been previously demonstrated that CLC-2 channels are involved in various cellular functions, including ion homeostasis, cell volume regulation, transepithelial transport, regulation of electrical excitability, cell migration and cell proliferation (30). Furthermore, CLC-2 channels have been indicated to be involved in the cell proliferation and invasive cell migration of primary brain tumors cells, particularly human glioma cells (31). As the majority of primary brain tumors arise from glial cells, glioma cell migration resembles progenitor cell migration during embryonic brain development $(32,33)$. In the CNS, OLs are responsible for the formation of myelin, and OLs are produced following OPC differentiation and maturation $(7,34)$. The present study demonstrated that OPCs successfully differentiated into mature OLs with T3 supplement; however, inhibition of CLC-2 channels repressed OPCs differentiation, which was 
A
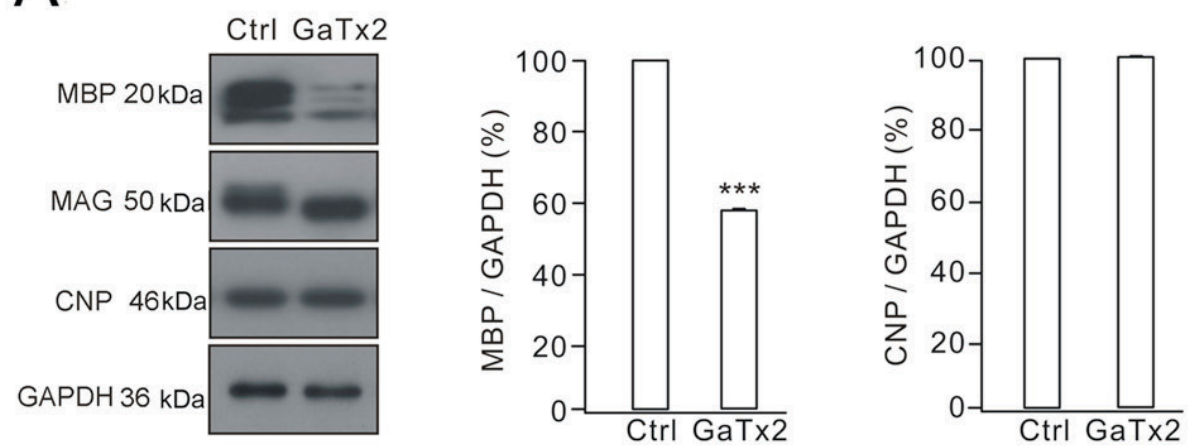

B
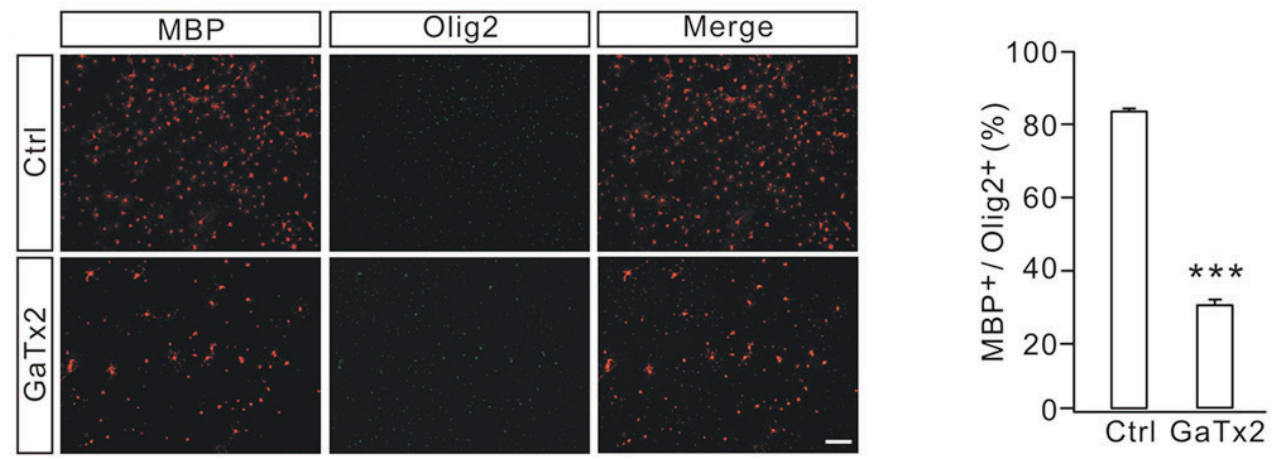

\section{C}
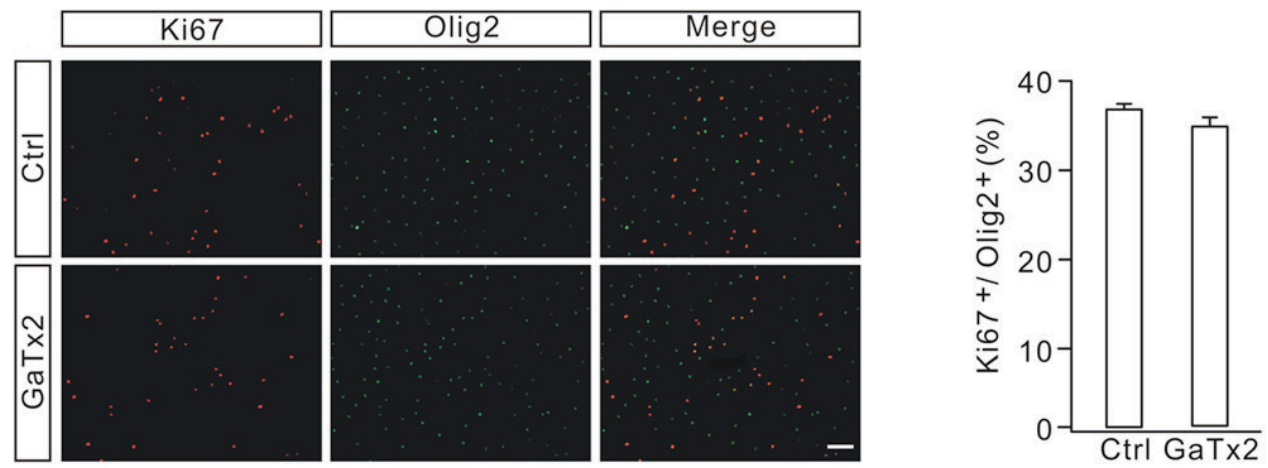

Figure 4. Effects of GaTx2 on OL differentiation. (A) MBP, MAG and CNP protein expression levels were assessed by western blotting. Control cells were treated with PDGF-AA for 1 day and T3 was added in for 3 days. In the experimental group, cells were treated with T3 together with GaTx2 ( $2 \mathrm{nM}$ for $72 \mathrm{~h}$ ). (B) Immunocytochemical staining of MBP and Olig2 indicated that GaTx2 may inhibit OL differentiation. (C) Immunocytochemical staining of Ki67 indicated that GaTx2 had no effect on OPC proliferation. The experiment was repeated four times. Scale bar, $40 \mu \mathrm{m} .{ }^{* * * *} \mathrm{P}<0.001$ vs. Ctrl. OLs, oligodendrocytes; MBP, myelin basic protein; MAG, myelin-associated glycoprotein; CNP, 2',3'-cyclic nucleotide 3'-phosphodiesterase; T3, triiodothyronine; Olig2, oligodendrocyte transcription factor 2; Ki67, marker of proliferation Ki67; OPCs, oligodendrocyte precursor cells; Ctrl, control.

consistent with a previous report where mice lacking CLC-2 $\left(\mathrm{Clcn}^{-/-}\right.$mice) exhibited abnormal morphology of the white matter and resembled the pathology of patients with megalencephalic leukoencephalopathy with subcortical cysts (35). By contrast, the excessive activation of CLC-2 may lead to cell damage. A report concerning the white matter of neonatal rats demonstrated that ischemia-hypoxia elevated the activation of CLC-2 and subsequently initiated apoptosis in OLs (23). He et al (36) reported that, in the white matter of newborn rats that presented with gestational diabetes mellitus, activation of CLC-2 resulted in apoptosis and myelination inhibition via repression of the phosphatidylinositol 3-kinase-protein kinase B signaling pathway. DIDS was demonstrated to prevent high glucose-induced damage to white matter damage, which may be due to the fact that following the differentiation of OPCs into early OLs, these immature OLs are susceptible to injury factors, leading to excessive activation of CLC-2 channels and enhanced $\mathrm{Cl}^{-}$currents (36). 
A

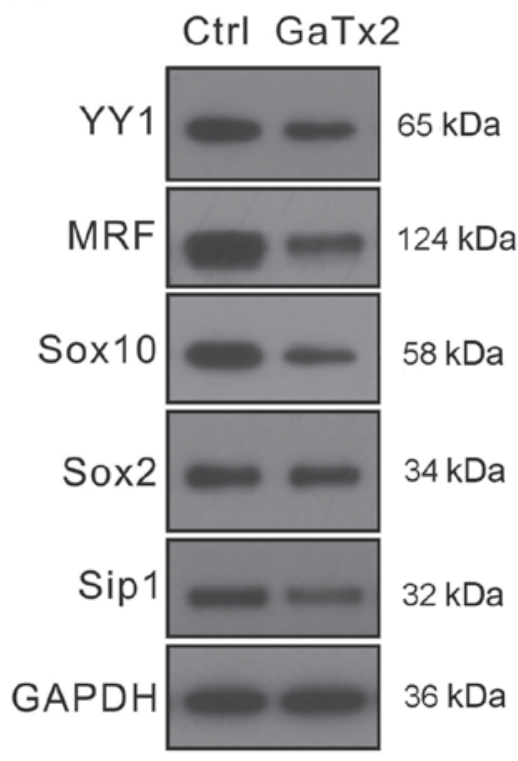

B

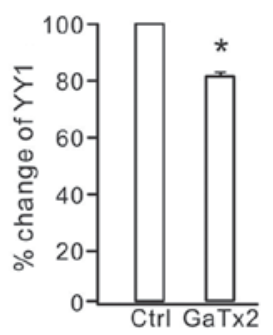

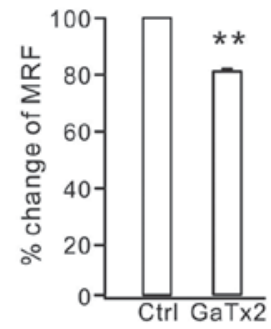

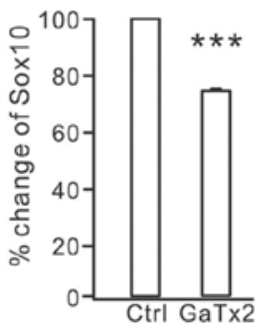

Figure 5. CLC-2 inhibition altered the protein expression levels of transcription factors involved in OL differentiation. (A) Protein expression levels of the transcription factors YY1, MRF, Sip1, Sox 2 and Sox10 were measured in control and GaTx2 groups by western blotting. (B) Densitometric analysis was performed to quantify the protein levels following western blotting. Levels of transcription factors in the control were set at $100 \%$ and the corresponding percentage changes of these proteins are presented. The experiment was repeated three times. ${ }^{*} \mathrm{P}<0.05,{ }^{* *} \mathrm{P}<0.01$ and ${ }^{* * *} \mathrm{P}<0.001$ vs. Ctrl. CLC-2, voltage-gated chloride channel 2; OLs, oligodendrocytes; YY1, yin yang 1; MRF, myelin regulatory factor; Sip1, Smad-interacting protein 1; Sox, sex-determining region Y-box; Ctrl, control.

It has been demonstrated that altered expression of CLC-2 is associated with alterations in $\mathrm{Cl}^{-}$currents (37). In vitro single cell recordings indicated that high concentrations of glucose led to enhanced opening of CLC-2 on cell membranes $(38,39)$. In the present study, the patch-clamp recordings demonstrated that $\mathrm{Cl}^{-}$currents in OLs were higher compared with those in OPCs, indicating that CLC-2 may serve roles in OL differentiation. Recordings from mouse astrocytes demonstrated that CLC-2-mediated current density was significantly lower in astrocytes isolated from immature brain and in reactive astrocytes within a lesion site but functional expressed in mature astrocytes isolated from adult mouse brain, indicating that as in oligodendrocytes, CLC-2 channels also served a role in astrocyte development and differentiation (40). In neurons, CLC-2 channels were reported to potentially constitute part of the background conductance serving as a safeguard mechanism to prevent chloride accumulation at synapses (41). It may be hypothesized that CLC-2 channels may have additional functions in mature OLs that do not occur in undifferentiated OPCs. Therefore, the role of CLC-2 in glial cells requires further investigation.

A previous study has demonstrated that the differentiation of OPCs is regulated by the induction of several transcription factors, most notably Sox10, Olig2, Sip1, MRF and YY1 (7), which are present in OPCs and in post-mitotic OLs, while Sox 2 has been reported to function in the maintenance of an undifferentiated state and the repression of myelin gene expression in OPCs, which is consistent with the results of the present study. Specifically, in the current study, the inhibition of CLC-2 channels led to reduced expression levels of Sox10, Sip1, MRF and YY1, while the expression levels of Sox2, a transcriptional repressor, were unchanged.
Based on these results, enhancing the expression of positive transcription factors may be a major role of CLC-2 channels in OPC differentiation. However, the mechanism underlying the regulatory effect of CLC-2 on these transcription factors requires further investigation.

In conclusion, the present study demonstrated that voltage-gated CLC-2 was associated with OPC differentiation and myelin gene transcription factors during development. The effect of CLC-2 inhibition on OPC differentiation by its specific inhibitor GaTx2 was not a result of altered proliferation. Furthermore, CLC-2 inhibition reduced the expression of various myelin protein transcription factors, including YY1, MRF, Sox10 and Sip1. Taken together, the results of the current study indicated that CLC-2 may serve as an important positive regulator in OPC differentiation by regulating the expression of myelin gene transcription factors. The present study only performed in vitro experiments on cells. In vivo studies on animals are required to demonstrate the effects of CLC- 2 on myelin development, injuries and repair. This will provide the clinical target for myelin-related diseases.

\section{Acknowledgements}

The present study was supported by grants from the National Key Research and Development Program of Ministry of Technology and Science of China (grant no.2017YFA0104200), the National Natural Science Foundation of China (grant nos. 81460182, 31460257, 81571098, 31260243 and 31560273), the Ningixa 13th Plan of Five-Year Major Scientific Program (grant no. 2016BZ 07) and Western First-Class Construction Disciplines in Basic Medical Sciences, Ningxia Medical University (grant no. NXYLXK2017B07). 


\section{References}

1. Tauheed AM, Ayo JO and Kawu MU: Regulation of oligodendrocyte differentiation: Insights and approaches for the management of neurodegenerative disease. Pathophysiology 23: 203-210, 2016.

2. Mi S, Lee X, Hu Y, Ji B, Shao Z, Yang W, Huang G, Walus L, Rhodes K, Gong BJ, et al: Death receptor 6 negatively regulates oligodendrocyte survival, maturation and myelinatin. Nat Med 17: 816-821, 2011.

3. Wolswijk G: Oligodendrocyte survival, loss and birth in lesions of chronic-stage multiple sclerosis. Brain 123: 105-115, 2000

4. Billiards SS, Haynes RL, Folkerth RD, Borenstein NS Trachtenberg FL, Rowitch DH, Ligon KL, Volpe JJ and Kinney HC: Myelin abnormalities without oligodendrocyte loss in periventricular leukomalacia. Brain Pathol 18: 153-163, 2008.

5. Boespflug-Tanguy O, Labauge P, Fogli A and Vaurs-Barriere $\mathrm{C}$ : Genes involved in leukodystrophies: A glance at glial functions. Curr Neurol Neurosci Rep 8: 217-229, 2008.

6. Miller RH: Regulation of oligodendrocyte development in the vertebrate CNS. Prog Neurobiol 67: 451-467, 2002.

7. Emery B: Regulation of oligodendrocyte differentiation and myelination. Science 330: 779-782, 2010.

8. Wang S, Sdrulla AD, diSibio G, Bush G, Nofziger D, Hicks C, Weinmaster $\mathrm{G}$ and Barres BA: Notch receptor activation inhibits oligodendrocyte differentiation. Neuron 21: 63-75, 1998.

9. Charles P, Hernandez MP, Stankoff B, Aigrot MS, Colin C, Rougon G,Zalc B and Lubetzki C: Negative regulation of central nervous system myelination by polysialylated-neural cell adhesion molecule. Proc Natl Acad Sci USA 97: 7585-7590, 2000

10. Mi S, Miller RH, Lee X, Scott ML, Shulag-Morskaya S, Shao Z, Chang J, Thill G, Levesque M, Zhang M, et al: LINGO-1 negatively regulates myelination by oligodendrocytes. Nat Neurosci 8 : 745-751, 2005

11. Arnett HA, Fancy SP, Alberta JA, Zhao C, Plant SR, Kaing S, Raine CS, Rowitch DH, Franklin RJ and Stiles CD: bHLH transcription factor Olig1 is required to repair demyelinated lesions in the CNS. Science 306: 2111-2115, 2004

12. Fu H, Qi Y, Tan M, Cai J, Takebayashi H, Nakafuku M, Richardson W and Qiu M: Dual origin of spinal oligodendrocyte progenitors and evidence for the cooperative role of Olig2 and $\mathrm{Nkx} 2.2$ in the control of oligodendrocyte differentiation. Development 129: 681-693, 2002.

13. Emery B, Agalliu D, Cahoy JD, Watkins TA, Dugas JC, Mulinyawe SB, Ibrahim A, Ligon KL, Rowitch DH and Barres BA: Myelin gene regulatory factor is a critical transcriptional regulator required for CNS myelination. Cell 138: 172-185, 2009.

14. Liu Z,Hu X, Cai J, Liu B, Peng X, Wegner M and Qiu M: Induction of oligodendrocyte differentiation by Olig2 and Sox 10: Evidence for reciprocal interactions and dosage-dependent mechanisms. Dev Biol 302: 683-693, 2007.

15. He Y, Dupree J, Wang J, Sandoval J, Li J, Liu H, Shi Y, Nave KA and Casaccia-Bonnefil P: The transcription factor Yin Yang 1 is essential for oligodendrocyte progenitor differentiation. Neuron 55: 217-230, 2007.

16. Wegner M: A matter of identity: Transcriptional control in oligodendrocytes. J Mol Neurosci 35: 3-12, 2008.

17. Fancy SP, Baranzini SE, Zhao C, Yuk DI, Irvine KA, Kaing S, Sanai N, Franklin RJ and Rowitch DH: Dysregulation of the Wnt pathway inhibits timely myelination and remyelination in the mammalian CNS. Genes Dev 23: 1571-1585, 2009.

18. Zhou L, Shao CY, Xu SM, Ma J, Xie YJ, Zhou L, Teng P, Wang Y, Qiu M and Shen Y: GSK3 $\beta$ promotes the differentiation of oligodendrocyte precursor cells via $\beta$-catenin-mediated transcriptional regulation. Mol Neurobiol 50: 507-519, 2014.

19. Williamson AV, Compston DA and Randall AD: Analysis of the ion channel complement of the rat oligodendrocyte progenitor in a commonly studied in vitro preparation. Eur J Neurosci 9: 706-720, 1997.

20. Kraus V, Srivastava R, Kalluri SR, Seidel U, Schuelke M, Schimmel M, Rostasy K, Leiz S, Hosie S, Grummel V and Hemmer B: Potassium channel KIR4.1-specific antibodies in children with acquired demyelinating CNS disease. Neurology 82: 470-473, 2014

21. Srivastava R, Aslam M, Kalluri SR, Schirmer L, Buck D, Tackenberg B, Rothhammer V, Chan A, Gold R, Berthele A, et al: Potassium channel KIR4.1 as an immune target in multiple sclerosis. N Engl J Med 367: 115-123, 2012.
22. Kirischuk S, Scherer J, Möller T, Verkhratsky A and Kettenmann H: Subcellular heterogeneity of voltage-gated Ca2+ channels in cells of the oligodendrocyte lineage. Glia 13: 1-12, 1995.

23. Zhao B, Quan H, Ma T, Tian Y, Cai Q and Li H: 4,4'-Diisothiocyanostilbene-2,2'-disulfonic acid (DIDS) ameliorates ischemia-hypoxia-induced white matter damage in neonatal rats through inhibition of the voltage-gated chloride channel ClC-2. Int J Mol Sci 16: 10457-10469, 2015.

24. Yang $Z$, Watanabe $M$ and Nishiyama A: Optimization of oligodendrocyte progenitor cell culture method for enhanced survival. J Neurosci Methods 149: 50-56, 2005.

25. Wang Z, Wang YN, Sun CL, Yang D, Su LD, Xie YJ, Zhou L, Wang Y and Shen Y: C-terminal domain of ICA69 interacts with PICK1 and acts on trafficking of PICK1-PKC $\alpha$ complex and cerebellar plasticity. PLoS One 8: e83862, 2013.

26. Lalor PA, Mapp PI, Hall PA and Revell PA: Proliferative activity of cells in the synovium as demonstrated by a monoclonal antibody, Ki67. Rheumatol Int 7: 183-186, 1987.

27. Thiemann A, Gründer S, Pusch M and Jentsch TJ: A chloride channel widely expressed in epithelial and non-epithelial cells. Nature 356: 57-60, 1992.

28. Sík A, Smith RL and Freund TF: Distribution of chloride channel-2-immunoreactive neuronal and astrocytic processes in the hippocampus. Neuroscience 101: 51-65, 2000.

29. Weng Q, Chen Y, Wang H, Xu X, Yang B, He Q, Shou W, Chen Y, Higashi Y, van den Berghe V, et al: Dual-mode modulation of Smad signaling by Smad-interacting protein Sip1 is required for myelination in the central nervous system. Neuron 73: 713-728, 2012.

30. Jentsch TJ, Stein V, Weinreich F and Zdebik AA: Molecular structure and physiological function of chloride channels. Physiol Rev 82: 503-568, 2002

31. Olsen ML, Schade S, Lyons SA, Amaral MD and Sontheimer H: Expression of voltage-gated chloride channels in human glioma cells. J Neurosci 23: 5572-5582, 2003.

32. Levison SW, Chuang C, Abramson BJ and Goldman JE: The migrational patterns and developmental fates of glial precursors in the rat subventricular zone are temporally regulated. Development 119: 611-622, 1993.

33. Amberger VR, Avellana-Adalid V, Hensel T, Baron-Van Evercooren A and Schwab ME: Oligodendrocyte-type 2 astrocyte progenitors use ametalloendoprotease to spread and migrate on CNS myelin. Eur J Neurosci 9: 151-162, 1997.

34. Huang H, Zhao XF, Zheng K and Qiu M: Regulation of the timing of oligodendrocyte differentiation: Mechanisms and perspectives. Neurosci Bull 29: 155-164, 2013.

35. Blanz J, Schweizer M, Auberson M, Maier H, Muenscher A, Hübner CA and Jentsch TJ: Leukoencephalopathy upon disruption of the chloride channel ClC-2. J Neurosci 27 6581-6589, 2007.

36. He F, Peng Y, Yang Z, Ge Z, Tian Y, Ma T and Li H: Activated $\mathrm{ClC}-2$ inhibits p-Akt to repress myelination in GDM newborn rats. Int J Biol Sci 13: 179-188, 2017.

37. Guo R, Pan F, Tian Y, Li H, Li S and Cao C: Down-regulation of ClC-3 expression reduces epidermal stem cell migration by inhibiting volume-activated chloride currents. J Membr Biol 249: 281-292, 2016

38. Klaus F, Laufer J, Czarkowski K, Strutz-Seebohm N, Seebohm G and Lang F: PIKfyve-dependent regulation of the Cl- channel ClC-2. Biochem Biophys Res Commun 381: 407-411, 2009.

39. Palmada M, Dieter M, Boehmer C, Waldegger S and Lang F: Serum and glucocorticoid inducible kinases functionally regulate ClC-2 channels. Biochem Biophys Res Commun 321: 1001-1006, 2004.

40. Makara JK, Rappert A, Matthias K, Steinhäuser C, Spät A and Kettenmann H: Astrocytes from mouse brain slices express $\mathrm{ClC}$-2-mediated $\mathrm{Cl}$ - currents regulated during development and after injury. Mol Cell Neurosci 23: 521-530, 2003.

41. Földy C, Lee SH, Morgan RJ and Soltesz I: Regulation of fast-spiking basket cell synapses by the chloride channel ClC-2 Nat Neurosci 13: 1047-1049, 2010.

This work is licensed under a Creative Commons Attribution-NonCommercial-NoDerivatives 4.0 International (CC BY-NC-ND 4.0) License. 